$\mathrm{TM}-53$

\title{
OFFSETTING OPERATIONS IN SOLID MODELLING
}

\author{
by \\ Jarosław R. Rossignac \\ Aristides A. G. Requicha \\ PRODUCTION AUTOMATION PROJECT
}

June 1985 

Technical Memorandum No. 53

\author{
PRODUCTION AUTOMATION PROJECT \\ Department of Electrical Engineering \\ College of Engineering and Applied Science \\ University of Rochester \\ Rochester, New York 14627
}

TM-53

OFFSETTING OPERATIONS IN SOLID MODELLING

by

Jarosław R. Rossignac

Aristides A. G. Requicha

June 1985

Keywords: Approximations of curves and surfaces, CAD/CAM, computational geometry, constructive solid geometry, geometric modelling, offsets.

The work reported in this paper was supported by the National Science Foundation under Computer Engineering Grants ECS-81-04646 and ECS-84-03882, and by companies in the Production Automation Project's Industrial Associates program. Any opinions, findings, conclusions or recommendations expressed or implied are those of the authors and do not necessarily reflect the views of the N.S.F. or the Industrial Associates of the P.A.P. The paper is based in part on Rossignac's Ph.D. Dissertation submitted to the Electrical Engineering Department of the University of Rochester. 



\begin{abstract}
The range of operations on solids supported by current geometric modelling systems is very limited. Typically, solids represented in a modeller can be transformed by rigid motions and combined by Boolean operations. This paper introduces another family of transformations, called solid offsetting, which map solids into solids.

Offset solids are expanded or contracted versions of an original object. Offsetting operations are potentially useful for tolerance analysis, clearance testing, design-rule checking in VLSI, modelling of etching and coating processes, cutter path generation for numerically-controlled machine tools, collision free path planning for robot motions, and for constant-radius rounding and filleting ("blending") of solids.

This paper discusses mathematical properties of solid offsetting, associated representations and algorithms, support of offsetting operations in solid modellers, and applications. Results of an experimental implementation are presented.
\end{abstract}





\section{TABLE OF CONTENTS}

1. Introduction

2. Mathematical definitions and properties of offsets

2.1 Definitions

2.2 Equality and inclusion

2.3 Closure under offsetting operations

2.4 Rigid motions and Boolean operations

2.5 Successive offsetting

2.6 Boundaries and n-offsets

3. Computational requirements

3.1 Representations

3.2 Algorithms

4. Boundary evaluation

4.1 Tentative face generation

4.2 Surface/surface intersection

4.3 Classification procedures

4.3.1 Curve/solid classification

4.3.2 Point/solid classification

4.3.3 Distance between a point and a solid

4.3.4 Neighborhoods

4.4 Simplified BReps

5. An experimental implementation

6. Applications

6.1 Blending

6.2 Other applications

7. Summary and conclusions

References 



\section{INTRODUCTION}

Solid modelling theory and technology are becoming increasingly well understood, and their commercial and industrial exploitation is progressing rapidly [Requicha \& Voelcker 82, 83]. However, the range of operations on solids supported by current modellers is very limited. Typically, solids represented in a modeller can be transformed by rigid motions, which are straightforward and well-known in computer graphics [Newman \& Sproull 79 , Foley \& van Dam 82], and can be combined by Boolean operations, which are complex but important [Requicha \& Voelcker 85]. Initial results of research aimed at modelling bending operations have been reported recently [Fournier \& Wesley 82, Barr 84].

This paper introduces a new family of transformations, called solid offsetting (abbreviated $s$-offsetting), that map solids into solids. We discuss mathematical properties of solid offsetting, representations and algorithms to support offsetting operations in solid modellers, applications, and experimental results.

Offset solids are expanded or contracted versions of an original object. To s-offset a solid $S$ by a positive distance $r$ one adds to the solid all the points exterior to $S$ that lie within a distance $r$ of the boundary of $S$. For a negative s-offset one subtracts from the solid all the points of $S$ within a distance $r$ from its boundary. (Formal definitions are given in Section 2 below.) Positive and negative s-offsets for a simple L-shaped " $2-\mathrm{D}$ solid" are shown in Figure 1.

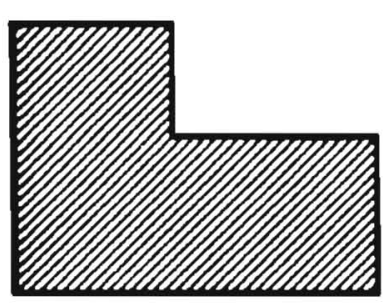

(a)

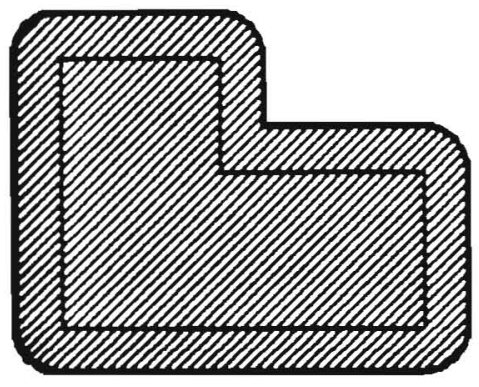

(b)

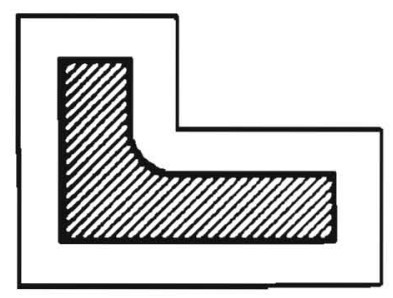

(c)

Figure 1

A simple L-shaped object (a), a positive offset (b), and a negative offset (c). 
Solid offsetting appears to be very useful. Potential applications are summarized in Section 6 below. They cover a wide range, from tolerance analysis and clearance testing, through modelling of such physical processes as coating or etching, to rounding and filleting - notice the vertex rounding effects in Figures 1b-c.

Operations related to solid offsetting have been used to calculate mass properties of solids [Lee and Requicha $82 \mathrm{~b}$ ], to plan collision-free paths for robots [Lozano-Perez \& Wesley 79], to check design rules in VLSI [Barton $\&$ Buchanan 80], and to generate cutter paths for numerically-controlled (NC) machine tools [Pressman \& Williams 77, Faux \& Pratt 79]. In [Lee and Requicha $82 \mathrm{~b}$ ] the primitives in a solid's constructive solid geometry (CSG) representation [Requicha 80] are expanded and contracted to reduce set inclusion and disjointness tests to point membership classification, which determines whether a point is inside, on the boundary of, or outside a solid. In [Lozano-Perez \& Wesley 79] obstacles are expanded, again to reduce solid interference tests to point membership classification. 2-D objects are expanded and contracted in VLSI applications to detect violations of minimum-distance, maximum and minimum size, and nonoverlap rules. In $\mathrm{NC}$ and related applications one offsets smooth surfaces by "moving along the normal" by a distance $r$ to generate other surfaces, and offsets planar curves similarly to generate other curves. (We refer to this operation as normal offsetting or simply n-offsetting, to distinguish it from solid offsetting.) The current understanding of normal offsetting is summarized in [Tiller \& Hanson 84] and [Klass 83]. Briefly, there is no accepted mathematical definition of $\mathrm{n}$-offsetting for surfaces and curves that are only piecewise smooth, and even when surfaces and curves are smooth n-offsetting may lead to cusps and self-intersections. Heuristic approaches proposed in [Tiller \& Hanson 84] provide reasonable results in many but not all of the possible cases.

Solid offsetting operations are special cases of so-called Minkowski sums and differences. These are important in the field of geometric probability and have been studied extensively by the French school of "mathematical morphology" [Matheron 75, Serra 82], which is primarily motivated by problems of texture analysis for geological applications. A few of the properties presented below have been derived independently in the mathematical morphology work. 
The remainder of this paper is organized as follows. We begin in Section 2 with formal definitions and mathematical properties of s-offsets, and show that $\mathrm{n}$-offsets are useful to study the boundaries of offset solids. Sections 3 and 4 deal with representations and algorithms for offset solids. Section 5 discusses an experimental modeller, Section 6 addresses potential applications, and Section 7 summarizes the paper.

\section{MATHEMATICAL DEFINITIONS AND PROPERTIES OF OFFSETS}

In this section we define offsets, list some of their mathematical properties, and discuss the practical importance of these properties. Proofs for most of the results are omitted. They can be found in [Rossignac 85] and involve elementary point-set topology [Mendelson 75].

\subsection{Definitions}

We model physical solids by r-sets, which are bounded, regular, and semi-analytic subsets of 3-D Euclidean space $\left(E^{3}\right)$ [Requicha 77, 80]. (Recall that a set $S$ is regular if $S=k i S$, where $k$ and $i$ denote, respectively, topological closure and interior.) The (regularized) positive solid offset of a regular set $S$ by a positive distance $r$ is

$$
S \uparrow^{*} r=\{\mathbf{p}: \exists \mathbf{q} \in S,\|\mathbf{p}-\mathbf{q}\| \leq r\} .
$$

Note that when $S$ is empty $S \uparrow^{*} r$ is also empty. We refer to positive soffsetting also as growing or expanding. (Non-regularized positive offsets can also be defined [Rossignac 85], and are called generalized balls in the mathematical literature [Nadler 78]; offsetting operations can also be applied to non-regular sets [Rossignac 85]. Offsets discussed in this paper are assumed to be regularized.)

An equivalent definition is

$$
S \uparrow^{*} r=\bigcup_{\mathbf{p} \in S} B^{*}(\mathbf{p}, r),
$$

where $B^{*}(\mathbf{p}, r)=\{\mathbf{q}:\|\mathbf{q}-\mathbf{p}\| \leq r\}$ is a closed ball of radius $r$ centered at $\mathbf{p}$. This alternative definition shows that one can think of $S \uparrow^{*} r$ as the volume 
swept by a solid sphere of radius $r$ as its center moves throughout the set $S$.

There is yet another equivalent definition in terms of point/set distances. First recall that the distance of a point $\mathbf{p}$ to a set $S$ is the minimal distance between $\mathbf{p}$ and points of $S$. More precisely, it is defined as [Nadler $78]$

$$
d(\mathbf{p}, S)=\inf _{\mathbf{q} \in S}\|\mathbf{p}-\mathbf{q}\| .
$$

When $S$ is regular, and hence closed, the greatest lower bound is attained for at least one closest point $\mathbf{q}$ of $S$, and therefore one can replace the infimum with minimum in the definition. There may be several closest points. If $p$ is a point of $S$ then $d(\mathbf{p}, S)=0$, and if $\mathbf{p}$ is exterior to $S$ then the closest points $\mathbf{q}$ lie in $\partial S$, the topological boundary of $S$.

The positive offset of a regular and non-empty $S$ can also be defined as

$$
\left.S \uparrow^{*} r=\{\mathrm{p}: d(\mathrm{p}, S) \leq r)\right\} .
$$

Thus, to expand $S$ one adds to it all the points whose distances to $S$ do not exceed $r$.

The (regularized) negative solid offset of a non-empty $S$ is defined as the complement of the expanded complement. More precisely,

$$
S \downarrow^{*} r=c^{*}\left(\left(c^{*} S\right) \uparrow^{*} r\right),
$$

where $c^{*}$ denotes regularized complement [Requicha 77,80$]$. We refer to negative $\mathrm{s}$-offsets also as contracted or shrunk solids.

\subsection{Equality and inclusion}

It follows immediately from the definitions that if two sets $A$ and $B$ are equal so are their s-offsets by $r$, i.e., $A \uparrow^{*} r=B \uparrow^{*} r$ and $A \downarrow^{*} r=B \downarrow^{*} r$. Note, however, that generally neither $A \uparrow^{*} r=B \uparrow^{*} r$ nor $A \downarrow^{*} r=B \downarrow^{*} r$ imply $A=B$.

Offsetting preserves inclusion relations. Thus, if $A \subset B$ then $A^{\uparrow^{*}} r \subset$ $B \uparrow^{*} r$ and $A \downarrow^{*} r \subset B \downarrow^{*} r$. 


\subsection{Closure under offsetting operations}

If $S$ is regular then its solid offsets $S \uparrow^{*} r$ and $S \downarrow^{*} r$ are also regular. Therefore regular sets are algebraically closed under offsetting operations. Offsetting by a finite $r$ clearly preserves boundedness, and we conjecture that it also preserves semi-analyticity, but we have no formal proof.

Algebraic closure of $r$-sets under offsetting is important. It implies that one can add offsetting operations to a modelling system that supports rigid motions and regularized Boolean operations, and be sure that the resulting sets are valid solids, and therefore can be used in the system as inputs for further operations.

\subsection{Rigid motions and Boolean operations}

Regularized intersection, union, difference, and complement, denoted respectively $\cap^{*}, \bigcup^{*},-^{*}, c^{*}$, are modified versions of their standard settheoretic counterparts [Requicha 77, 80]. In this paper regularized set operations on solids are called simply Boolean operations.

Rigid motions and Boolean operations are the fundamental means used in CSG for defining complex objects in terms of simpler, or primitive solids. It is tempting to s-offset solids defined by CSG by s-offsetting each primitive and combining the results, but it is easy to show by counterexamples that this procedure generally is incorrect.

Rigid motions and s-offsets commute since offsets are defined through distances, which are invariant under rigid motions.

The following properties summarize the relationships between offsetting and Boolean operations.

$$
\begin{gathered}
\left(c^{*} S\right) \uparrow^{*} r=c^{*}\left(S \downarrow^{*} r\right) \\
\left(c^{*} S\right) \downarrow^{*} r=c^{*}\left(S \uparrow^{*} r\right) \\
\left(A \bigcup^{*} B\right) \uparrow^{*} r=\left(A \uparrow^{*} r\right) \bigcup^{*}\left(B \uparrow^{*} r\right) \\
\left(A \bigcap^{*} B\right) \downarrow^{*} r=\left(A \downarrow^{*} r\right) \bigcap^{*}\left(B \downarrow^{*} r\right) \\
\left(A-^{*} B\right) \downarrow^{*} r=\left(A \downarrow^{*} r\right)-^{*}\left(B \uparrow^{*} r\right)
\end{gathered}
$$




$$
\begin{aligned}
& \left(A \bigcap^{*} B\right) \uparrow^{*} r \subset\left(A \uparrow^{*} r\right) \bigcap^{*}\left(B \uparrow^{*} r\right) \\
& \left(A \bigcup^{*} B\right) \downarrow^{*} r \supset\left(A \downarrow^{*} r\right) \bigcup^{*}\left(B \downarrow^{*} r\right)
\end{aligned}
$$

It follows from these properties that s-offsetting primitives in a CSG representation of $S$ and combining them yields $S \uparrow^{*} r$ only in very special cases. For example, expanding a solid whose CSG definition contains only unions can be done by expanding the primitives and unioning the results.

\subsection{Successive offsetting}

Growing operations are associative and commutative in the following sense:

$$
\left(S \uparrow^{*} a\right) \uparrow^{*} b=S \uparrow^{*}(a+b)=\left(S \uparrow^{*} b\right) \uparrow^{*} a
$$

Similar properties hold for shrinking operations:

$$
\left(S \downarrow^{*} a\right) \downarrow^{*} b=S \downarrow^{*}(a+b)=\left(S \downarrow^{*} b\right) \downarrow^{*} a
$$

However, a growing operation does not generally commute with a shrinking operation, and the two operations should not be viewed as "inverses" because

$$
\left(S \downarrow^{*} r\right) \uparrow^{*} r \subset S \subset\left(S \uparrow^{*} r\right) \downarrow^{*} r,
$$

but in general the inclusions are not equalities.

In fact, combinations of growing and shrinking operators are very useful, because of their rounding and filleting effects. We define rounding of $S$ by $r$ as

$$
R_{r}(S)=\left(S \downarrow^{*} r\right) \uparrow^{*} r,
$$

and filleting as

$$
F_{r}(S)=\left(S \uparrow^{*} r\right) \downarrow^{*} r .
$$

Figure 2 shows that $R_{r}$ rounds the convex edges and vertices of a solid, while $F_{r}$ fillets the concave edges and vertices. 


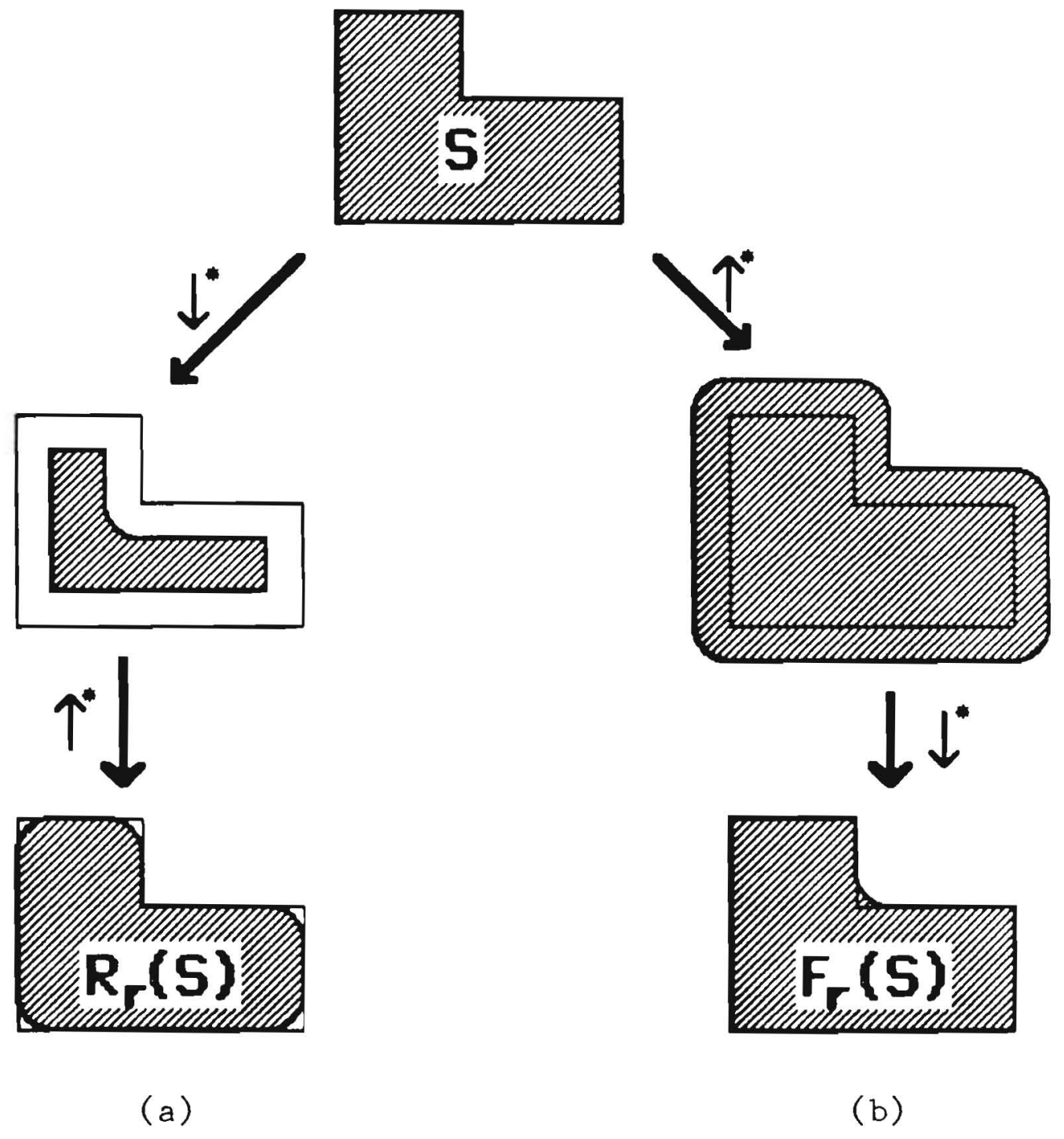

Figure 2

A set $S$ and its rounded (a) and filleted (b) versions. 


\subsection{Boundaries and $n-o f f s e t s$}

The topological boundary of an expanded solid $S \uparrow^{*} r$ is contained in the set of points that are at a distance $r$ from $S$, i.e.,

$$
\partial\left(S \uparrow^{*} r\right) \subset\{\mathbf{p}: d(\mathbf{p}, S)=r\}
$$

Figure 3 shows that the inclusion above generally is not an equality.

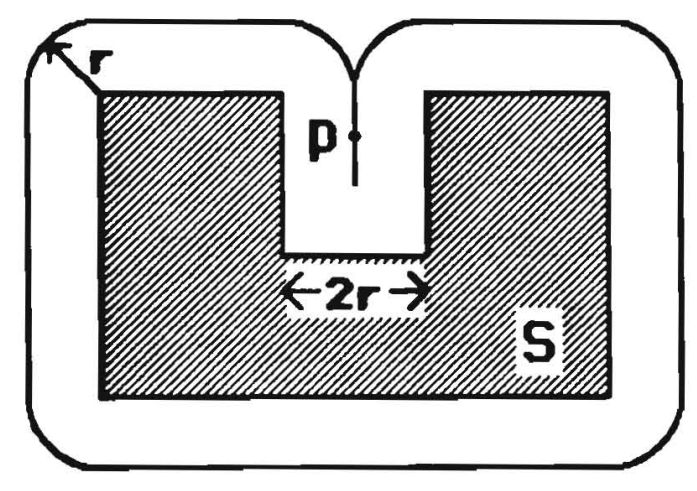

Figure 9

Point $\mathbf{p}$ is at distance $r$ from $S$ but is not on the boundary of $S \uparrow^{*} r$.

The analogous result for contracted solids is

$$
\partial\left(S \downarrow^{*} r\right) \subset\left\{\mathbf{p}: d\left(\mathbf{p}, c^{*} S\right)=r\right\} .
$$

One can also show that, for $\mathbf{p} \notin S, d(\mathbf{p}, S)=d(\mathbf{p}, \partial S)$. These properties are useful for constructing supersets of the boundaries of offset solids. Boundary supersets are important for boundary evaluation and other procedures discussed in Section 4.

The boundary of a solid (i.e., r-set) $S$ is a $G^{1}$ continuous surface (i.e., has continuous unit normal [Barsky \& DeRose 84]) except at singularities, which may be 1-dimensional (curves) or 0-dimensional (points). The singular curves are themselves $G^{1}$ continuous (i.e., have continuous unit tangents) except at singular points. For a (flat-faced) polyhedral solid $S$ the singular curves of $\partial S$ are the solid's edges, and the singular points the vertices. When $S$ is curved the singularities typically are a proper subset of the edges and vertices that appear in the solid's boundary representation. 
Let us construct a superset of $\partial\left(S \uparrow^{*} r\right)$. (Similar arguments apply to $S \downarrow^{*} r$.) First observe that, for $\mathbf{p} \notin S$, the distance $d(\mathbf{p}, S)$ must be attained for some point $\mathbf{q}$ of $\partial S$, and $\mathbf{q}$ must be in one of the following sets:

1) $F=\partial S-$ (all singularities);

2) $E=$ (singular curves) - (singular points);

3) $V=$ singular points.

We analyze each case separately. Suppose that $q \subset F$. Then, in a neighborhood of $\mathbf{q}$ there is a parameterization $\mathbf{q}=\mathbf{q}(u, v)$, and the distance $\|\mathbf{p}-\mathbf{q}\|$ must reach a minimum, which implies

$$
(\mathbf{p}-\mathbf{q}) \cdot \frac{\partial \mathbf{q}}{\partial u}=0 \quad \text { and } \quad(\mathbf{p}-\mathbf{q}) \cdot \frac{\partial \mathbf{q}}{\partial v}=0
$$

where the dot denotes inner product. Therefore the vector $\mathbf{p}-\mathbf{q}$ is normal to $F$ at $\mathbf{q}$ because $\frac{\partial \mathbf{q}}{\partial u}$ and $\frac{\partial \mathbf{q}}{\partial v}$ are tangent to the surface. (We assume regular parameterization and therefore $\frac{\partial \mathbf{q}}{\partial u}$ and $\frac{\partial \mathbf{q}}{\partial v}$ are not parallel.) Now construct the set $F \|_{r}^{+}$by displacing each point q of $F$ by a distance $r$ along the unit normal $\mathbf{n}$ at $\mathbf{q}$, i.e., let $\mathbf{p}=\mathbf{q}+\mathbf{r n} . F \|_{r}^{+}$is called the positive normal offset, (abbreviated positive n-offset) of $F$, and is the conventional "offset surface" used in NC applications, as noted in Section 1. It is clear from the construction that $F \|_{r}^{+}$includes all the points $\mathrm{p}$ for which the distance $d(\mathbf{p}, S)=r$ is attained for some $\mathbf{q}$ in $F$. Note, however, that not all points of $F \|_{r}^{+}$are at a distance $r$ from $S$; some may be at a smaller distance, as shown in Figure 4. Similar arguments show that $F \|_{r}^{-}$, the negative $\mathbf{n}^{-}$ offset of $F$, defined by displacing $\mathbf{q}$ inward by $r$, contains all points $\mathbf{p}$ at distance $r$ from the complement of $S$. N-offsets of smooth surfaces need not be smooth because they may self-intersect (see [Willmore 58, p. 117] for self-intersection conditions). In fact, $\mathrm{n}$-offsets of surfaces need not even be surfaces. For example, the negative n-offset by $r$ of a sphere of radius $r$ is a point - the center of the sphere. 


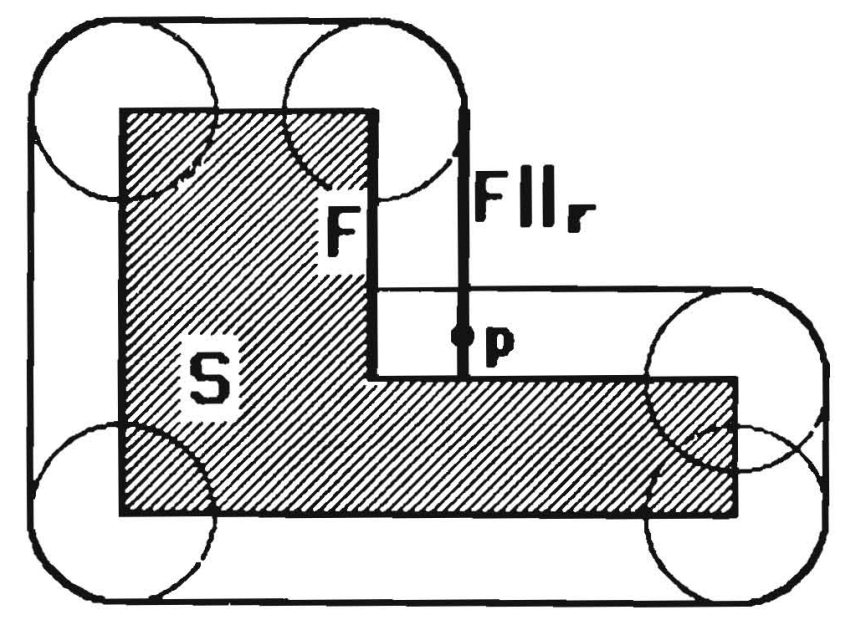

Figure 4

Point $\mathrm{p}$ is in the $\mathrm{n}$-offset by $r$ of the face $F$ of $S$ but its distance to $S$ is less than $r$.

Consider now $\mathrm{q} \in E$. Parameterizing the curve in a neighborhhod of $\mathbf{q}$ as $\mathbf{q}=\mathbf{q}(t)$ and differentiating the distance $\|\mathbf{p}-\mathbf{q}\|$ yields

$$
(\mathbf{p}-\mathbf{q}) \cdot \frac{\partial \mathbf{q}}{\partial t}=0
$$

Therefore $\mathbf{p}$ must lie in the normal plane to $E$ at $\mathbf{q}$ because $\frac{\partial q}{\partial t}$ is tangent to the curve. Since $\|\mathbf{p}-\mathbf{q}\|=r$, $\mathbf{p}$ lies in a circle of radius $r$ and center $\mathbf{q}$ in the normal plane. The set of points $\mathbf{p}$ on such circles for all $\mathbf{q}$ in $E$ is called the positive n-offset of $E$. (Negative n-offsets for curves are empty.) $E \|_{r}^{+}$can be viewed as the surface swept by a circle of radius $r$ when its center moves along the trajectory, or spine $E$. $\mathrm{N}$-offsets for curves are closely related to right circular constant generalized cylinders used in computer vision [Shafer \& Kanade 83], and were called canal or tubular surfaces in the mathematical literature of the turn of the century [Monge 1849, Salmon 1882], where they were defined in terms of envelopes of families of spherical surfaces.

Finally, if $\mathbf{q} \in V$ then $\mathbf{q}$ is an isolated point, and we define positive $n$-offset by $r$ of a point $q$ simply as a spherical surface of radius $r$ centered at q. (Negative $n$-offsets for points are empty.)

The set obtained by n-offsetting the $G^{1} 2-\mathrm{D}$ subsets of $\partial S$, the $G^{1} 1-\mathrm{D}$ subsets of its singular curves, its singular points, and unioning the results is 
simply called the $\mathrm{n}$-offset of $\partial S$. It follows from the previous discussion that the n-offset of $\partial S$ contains all points at distance $r$ from $S$, and therefore is a superset of the boundary of the offset solid $S \uparrow^{* *} r$.

The solids representable in most of the existent solid modellers must be bounded by planes, cylinders, cones, spheres - sometimes called the "natural quadrics", because they are easy to produce by conventional machining operations such as drilling and milling [Hakala et. al. 80] - and tori. (In the sequel we refer collectively to all these surfaces as standard surfaces.) Importantly, $\mathrm{n}$-offsetting a standard surface produces a surface of the same type. (Note, however, that $\mathrm{n}$-offsetting other quadrics, e.g. an ellipsoid, does not generally produce quadrics.) $\mathrm{N}$-offsetting an edge of intersection of two standard surfaces produces a canal surface, and, if this latter is smooth, $\mathrm{n}$-offsetting it produces another canal surface with the same spine. We conclude that to support offsetting operations in standard modellers only a new type of surface - a canal surface - must be introduced. The required canal surfaces, however, may be quite complicated because their spines may be the intersection of other canal surfaces, when offset solids are combined by Boolean operations and offset further. Closed form equations for canal surfaces can be obtained readily only in very simple special cases.

\section{COMPUTATIONAL REQUIREMENTS}

Our primary goal is to support offsetting operations in solid modellers that contain both CSG and boundary representations (BReps), and to ensure that offset solids are treated like other solids in the modeller, i.e., that they can be displayed, combined by Boolean operations, further offset, and so forth. This section discusses representational and algorithmic requirements for achieving this goal.

\subsection{Representations}

The (conceptually) simplest approach is to treat offsetting operations as "commands" that are entered by a user, executed, and discarded. Execution of the commands should produce both CSG and boundary representations for the offset solid that results from the operation. We investigated this approach and found that it is difficult to generate automatically CSG 
representations for offsets of complicated solids, and that the resulting representations are difficult to manipulate algorithmically. (CSG representations for offsets of simple solids, e.g. blocks, cylinders, spheres and cones, are easy to generate.)

We opted for an alternative approach, in which offset solids are represented by an extended form of CSG called CSG with offsetting, or simply CSGO. Representations in CSGO are trees containing offsetting operators as nonterminal nodes, in addition to the usual rigid motion and Boolean operators. Figure 5 shows a CSGO tree; by convention, the left subtree of a motion or offset node is a solid, and the right subtree contains the parameters that define the motion or the offset distance. What representations and algorithms are needed in a dual CSGO/BRep modeller?

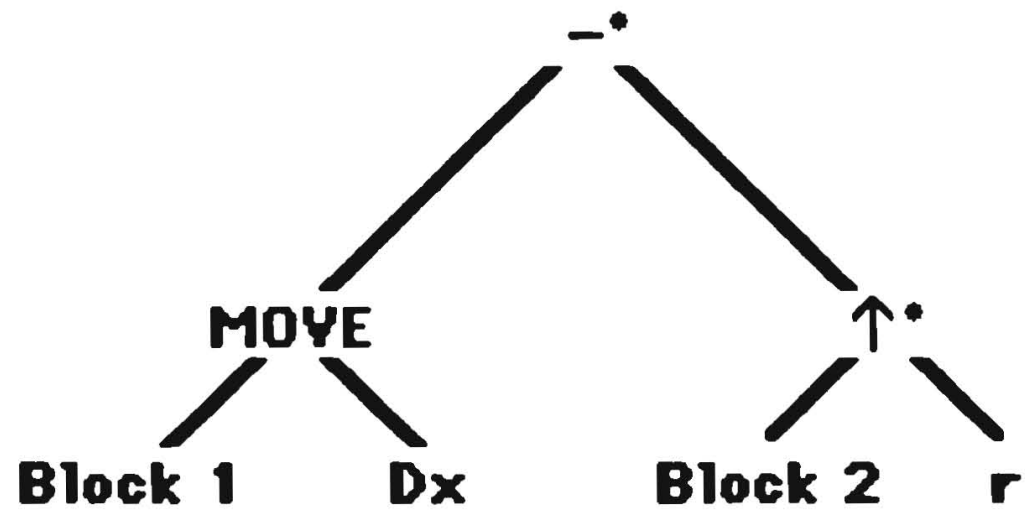

Figure 5

A CSGO tree.

CSGO trees are a trivial extension of the usual CSG trees, but BReps for offset solids are considerably more complex than those for solids bounded by the standard quadric and toroidal surfaces. Offsetting introduces new types of surfaces in a modeller - canal surfaces - and, since offset solids may be combined by Boolean operations, edges of intersection between canal surfaces or between canal and standard surfaces may also appear. Therefore we need representations for canal surfaces and for subsets of their intersection curves.

Canal surfaces can be represented indirectly, by their spine curves and radii, with the spine implicitly represented by two intersecting surfaces. (In 
essence, these are sweep representations [Requicha 80].) However, these representations are computationally awkward because they lead to a nightmare of indirections. Think, for example, of intersecting a curve with a canal surface represented by a radius and a spine, when the spine results from the intersection of two other canal surfaces, which in turn are represented indirectly, and so forth. Our approach is to represent canal surfaces indirectly (and exactly) but also carry an approximate representation, which is used in all the necessary numerical calculations.

The entire curves of intersection between two canal surfaces, or between a canal and a standard surface may be represented indirectly by using pointers to the surfaces. However, these curves need not be 1-manifolds (i.e., they may self-intersect) and therefore a subset of a curve cannot be represented simply by the host curve and a pair of endpoints [Requicha 80]. The standard representations for curve segments in solid modelling are based on parameterizations of the curves, but we do not know of any general methods for parameterizing the intersections of canal surfaces. These difficulties led us to represent edges through parameterized approximations. We also carry references to the intersecting surfaces, which may be used to refine the approximations when needed.

Edge approximation is a crucial issue in our approach. We devised a new approximation technique, called piecewise constant curvature approximation, which is especially well-suited for solid modelling [Rossignac 85, Rossignac \& Requicha 85]. Edges are approximated by piecewise circular curves, and the canal surfaces that result from n-offsetting such edges are approximated by smoothly joined pieces of tori and cylinders. The resulting curves have continuous unit tangents, and the surfaces have continuous unit normals, i.e., both are $G^{1}$ geometrically continuous [Barsky \& DeRose 84]. (In the sequel we use the abbreviation PCC both as a noun to denote "piecewise circular curve", and as an adjective to mean "piecewise constant curvature".)

In summary, our approach is to add offsetting nodes to CSG trees, and to represent in BReps the new surfaces and edges introduced by offsets both indirectly (exactly) and by PCC approximations. 


\subsection{Algorithms}

Today's solid modellers have facilities for generating calligraphic and shaded displays of the represented objects, for computing mass properties, and for evaluating the boundaries of objects defined by Boolean operations. What algorithms are needed to support such facilities in a CSGO/BRep system?

Calligraphic displays usually are generated in solid modellers by applying standard computer graphics techniques to edge lists, or to BReps. Shaded displays, however, often are generated directly from CSG by ray casting. The basic ray casting algorithm can be written in pseudo-code as follows [Roth 82]. (We use a pseudo-code based on the Algol/Pascal family of languages; procedures are assumed to return values.)

\section{ALGORITHM 1}

begin

for each Pixel in Screen

do begin

$E \leftarrow$ CreateRay (ViewPoint, Pixel);

$E w r t S \leftarrow$ ClassEdge $(E, S)$;

if $\operatorname{Ein} S \neq \emptyset$

then begin

$P \leftarrow$ FirstPoint $($ Ein $S)$;

$I \leftarrow \operatorname{CompIntensity~}(P$, LightSources $)$;

Display $(I)$;

end;

end.

end;

In words: cast a ray $E$ between the viewpoint and each pixel in the screen, find the first point $P$ where the ray enters the solid $S$, use an illumination model to compute the appropriate intensity $I$, and write it onto the screen. The essential procedure in this algorithm is ClassEdge, which takes an "edge" (i.e., a curve segment) $E$ and returns $E w r t S=$ (EinS, Eon $S$, Eout $S$ ), where Ein, Eon $S$, Eout $S$ are, respectively, the subsets of $E$ that are inside, on the boundary of, and outside the solid $S$ [Tilove 80, Requicha \& Voelcker 85]. 
There are many methods for computing mass properties of solids [Lee $\&$ Requicha $82 \mathrm{a}]$, but the most commonly used in dual-representation modellers are based on ray casting or cell classification [Lee \& Requicha $82 \mathrm{~b}$ ]. The ray casting algorithm involves the ClassEdge procedure mentioned above. Cell classification requires a procedure ClassPoint $(P, S)$, which determines whether a point $P$ is in the interior, boundary, or complement of a solid $S$.

Boundary evaluation is a considerably more complicated process than display or mass-property calculation. Again, there are many approaches to boundary evaluation, but several CSG/BRep modellers, including PADL-2, use a more elaborate version of the following basic algorithm.

\section{ALGORITHM 2}

begin

Generate a sufficient set of Tentative Faces;

Generate a sufficient set of Tentative Edges;

for each Tentative Edge $E$

$$
\begin{aligned}
& \text { do begin } \\
& \quad E w r t S \leftarrow \text { ClassEdge }(E, S) ; \\
& \text { AddToBRep }(E o n S) ; \\
& \text { end; }
\end{aligned}
$$

end.

Thus, one starts with tentative faces that are guaranteed to include the faces of the desired object. Then, pairwise intersection of tentative faces generates tentative edges, which are guaranteed to include those of the object. Extraneous portions of these tentative edges are discarded by classifying them with respect to the solid and retaining only those segments that are on the solid's boundary. (See [Requicha \& Voelcker 85] for details.)

The code fragment above may be imbedded in various boundary evaluation procedures - see e.g. [Tilove et al. 84]. The following is conceptually straightforward, and practically useful. It constructs incrementally a BRep for a solid $S$ represented by a CSG tree. BReps for the left and right (when applicable) subtrees of $S$ are assumed to have been previouly computed, by prior calls to BEval. 


\section{ALGORITHM 3}

procedure BEval $(S)$;

begin

case $S$ of

Primitive: BuildPrimBRep;

Motion: MoveBRep(S.Left);

Boolean: begin

TFaces $\leftarrow$ Faces of $S$.Left and $S$.Right;

for each Pair of TFaces $F, G$

do begin

$E \leftarrow F \cap G$;

Ewrt $S \leftarrow$ ClassEdge $(E, S)$;

AddToBRep(EonS);

end; $\{$ do $\}$

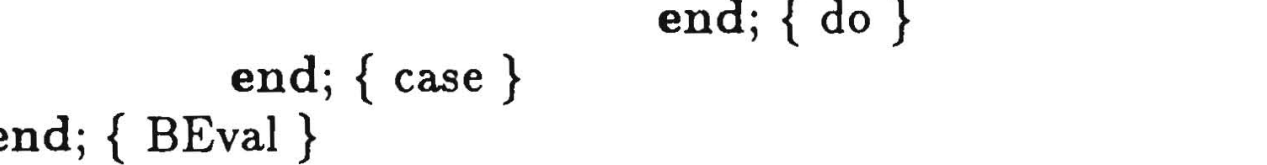

end; $\{$ case $\}$

end; $\{$ BEval $\}$

In words: look at the top node of the CSG tree of $S$; if $S$ is a primitive simply build its BRep; if it is a rigid motion node, apply the motion to the appropriate BRep; otherwise (the node is a Boolean operator) generate tentative faces by taking the faces of the two subtrees of $S$, and use Algorithm 2 .

Algorithm 3 can be readily modified for operation on CSGO representations. We need to add another branch to the case statement when the node corresponds to an offset operator. The new branch also will use Algorithm 2 , but we need a different way to generate tentative faces. We also need procedures to intersect these faces (some of which will lie in canal surfaces), and to classify edges with respect to solids represented in a CSGO/BRep dual scheme.

We conclude that the algorithmic requirements of boundary evaluation subsume those of display generation and mass property calculation. In essence they are: tentative face generation, surface/surface intersection, and edge classification. These three topics will be discussed in the next sections. 


\section{BOUNDARY EVALUATION}

This section discusses the main components of a boundary evaluation procedure for offset solids. As we saw earlier, the classification procedures required by boundary evaluation also provide means to generate displays and compute mass properties.

\subsection{Tentative face generation}

At each offset node in a CSGO tree we must generate a sufficient set of tentative faces, whose union is guaranteed to include the boundary of the offset object. This amounts to computing a superset of the boundary of the offset solid $S \uparrow^{*} r$ or $S \downarrow^{*} r$, which may be done by n-offsetting $\partial S$, as shown in Section 2.6. Therefore we must $\mathrm{n}$-offset the smooth faces, and singular curves and points of $\partial S$. The necessary information is contained in the BRep of $S$, and n-offsetting standard faces, canal faces, curves and points is straightforward.

One must ensure that the BReps used contain all the singularities. For example, the apex of a cone is a singular point and must be n-offset, but is not contained explicitly in many BRep schemes. (A convenient method for generating such isolated singular points in a BRep is to pass through them "dummy edges" [Rossignac 85].)

Note also that, instead of n-offsetting the actual faces of $S$, one can n-offset superfaces of $S$, i.e., 2-D sets that include the actual faces, and still produce a superset of the boundary of the offset solid. This is important because faces my be arbitrarily complex and therefore difficult to n-offset, while superfaces that are geometrically simple can be n-offset easily.

The following facts may be used to generate smaller supersets, and therefore to speed-up tentative face generation, as well as subsequent computations. (We assume below that the positive direction of the normal to the faces or superfaces of $S$ is toward the exterior of $S$.)

- Negative n-offsets (towards the interior of $S$ ) of faces need not be considered when computing tentative faces for $S \uparrow^{*} r$. Similarly, positive n-offsets of faces are not needed for $S \downarrow^{*} r$.

- Concave edges need not be n-offset for $S \uparrow^{*} r$. Similarly, convex edges are not needed for $S \downarrow^{*} r$. BRep edges that separate tangent faces 
are not singular curves, because the normal to $\partial S$ is continuous, and therefore never need to be n-offset.

\subsection{Surface/surface intersection}

Tentative edges are generated by pairwise intersection of tentative faces. If these lie in natural quadrics, the standard intersection routines of a solid modeller may be used; parameterizations for the resulting edges are readily available. But if the tentative faces are toroidal or are canal surfaces, approximate methods seem inevitable. Our approach has two steps: first we generate points on the intersection, and then we connect them by interpolatory PCCs. These two steps are explained in more detail below.

The curves of constant $u$ and constant $v$ in the "natural" parameterizations $F(u, v)$ of the standard surfaces are lines and circles, as shown in Figure 6. These lines and circles are called generators. To compute points in the intersection of two tentative faces we intersect a grid of $u$ and $v$ generators of one face with the other face. (We found that both $u$ and $v$ generators must be considered, otherwise intersections almost "parallel" to one of the families of generators may be missed entirely.) Because the generators are lines or circles, their intersection with standard surfaces can be computed by solving algebraic equations of degree at most four, and this can be done analytically [Rossignac 85]. At each point of the intersection we compute the normals to both faces. The cross product of the normals is the direction of the tangent to the intersection curve.
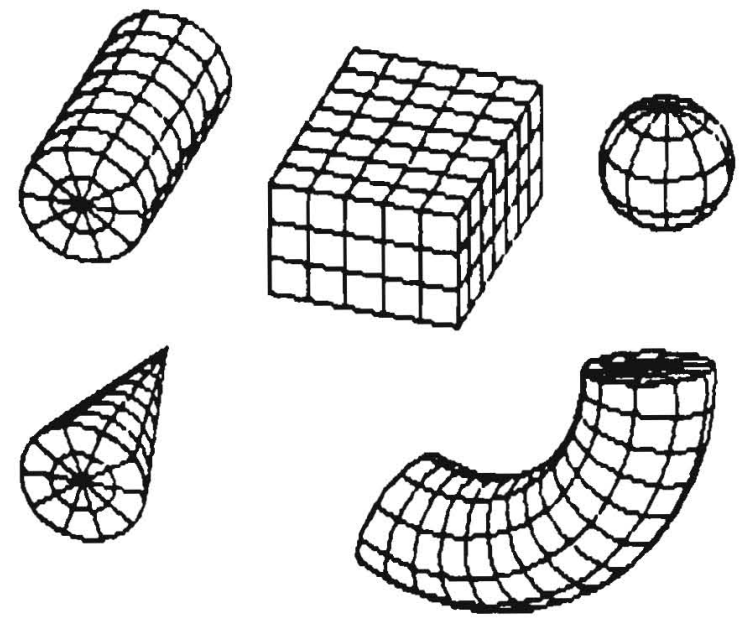

Figure 6

Standard primitives with generators. 
The result of the first step just described is a set of points and tangents. The second step is more delicate. It involves matching pairs of consecutive points along the curves and using the two points in each pair as endpoints for a span of a PCC approximation. Our matching algorithm is as follows. The intersections between the $u$ and $v$ generators of a face $F_{1}$ with a face $F_{2}$ are computed and stored. These intersection points lie in the boundaries of grid "cells" in $u, v$ space - see Figure 7 . We analyze each cell separately. If there are only two intersections in a cell's boundary we match them; otherwise we recursively subdivide the cell until each subcell contains only two intersections or a minimum resolution is reached. Matching at the lowest resolution level is done heuristically. It does not ensure that the topology of the intersection is correct, but such errors have never caused us any difficulties in our solid modelling work. (We experimented with various methods for matching points by using position and tangent information, but none of them were as reliable and efficient as the cell-based approach, which requires essentially no searching.) 
If $S$ is a primitive we use its real faces as superfaces, otherwise we use a set of tentative faces computed as explained in Section 4.1. The intersection of an edge $E$ with a superface $F$ in Algorithm 4 may be replaced with the intersection of $E$ and the host surface in which $F$ lies. Edge/surface intersection is a standard computation in solid modellers. It is usually accomplished by substituting the parametric definition $\mathbf{p}(t)$ of the edge in the implicit equation of the surface $f(\mathbf{p})=0$, and solving the resulting equation $f(\mathbf{p}(t))=0$ for $t$.

Procedure ClassEdge calls a point/solid classifier for midpoints of segments. Note that such points may lie in edges or faces of $S$, but cannot be vertices of $S$.

\subsubsection{Point/solid classification}

The standard recursive point classification algorithm for CSG [Requicha \& Voelcker 77] can be adapted to CSGO as follows.

\section{ALGORITHM 5}

procedure ClassPoint $(\mathbf{p}, S)$;

begin

case $S$ of

Primitive: ClassPoint WrtPrim $(\mathbf{p}, S)$;

Motion: ClassPoint $\left(M^{-1}(\mathbf{p}), S . L e f t\right) ;\{\operatorname{motion} M\}$

Boolean: Combine(ClassPoint(p, S.Left), ClassPoint(p,S.Right),S.Op);

Offset: ClassPointWrtOffset $(\mathbf{p}, S)$;

end;

$$
\text { end; }\{\text { case }\}
$$

Thus, we add to the case statement in the standard CSG classifier a branch to deal with offset operations. To classify a point $\mathrm{p}$ with respect to $S=X^{\uparrow^{*}} r$ we use the following procedure. (A similar procedure applies to $S=X \downarrow^{*} r$.) 


\section{ALGORITHM 6}

procedure ClassPointWrtOffset $(\mathbf{p}, S)$;

begin

$X \leftarrow S$.Left;

$r \leftarrow S$. Right; $\{$ Offset distance $\}$

if ClassPoint $(\mathbf{p}, X)=i n X$ or on $X$

then return in $S$

else case $\operatorname{Dist}(\mathbf{p}, \partial X)$ of

$<r$ : return in $S$;

$>r$ : return out $S$;

$=r:$ if $\operatorname{Nbhd}(\mathbf{p})=$ PartFull

then return on $S$

else return in $S$;

end $\{$ case $\}$;

end;

We need procedures to evaluate the distance between $\mathbf{p}$ and a solid's boundary, and to evaluate the neighborhood $\mathrm{Nbhd}(\mathbf{p})$ with respect to an offset solid $S$. These are discussed in the next two subsections.

\subsubsection{Distance between a point and a solid}

Algorithm 7 implements the method discussed in Section 2.6. It uses two auxiliary procedures ProjOnCurve and ProjOnSurf that compute normal projections, defined as follows. The normal projection set of point $\mathbf{p}$ on curve $C$ is the set of points $\mathbf{q}$ of $C$ for which the vector $\mathbf{p}-\mathbf{q}$ is normal to the tangent to $C$ at $\mathbf{q}$. Similarly, the normal projection set of $\mathbf{p}$ on surface $F$ is the set of points $\mathbf{q}$ of $F$ for which $\mathbf{p}-\mathbf{q}$ is normal to the tangent plane to $F$ at $q$. The theory of Section 2.6 implies that the normal projection set contains the point(s) of $C$ or $F$ for which the minimal distance to $\mathrm{p}$ is achieved. But it may also contain local minima and maxima of the distance. 


\section{ALGORITHM 7}

procedure $\operatorname{Dist}(\mathbf{p}, \partial S)$

begin

Dmin $\leftarrow \emptyset$;

for each singular point $\mathbf{q}$ of $\partial S$ do $D \min \leftarrow \min (D \min ,\|\mathbf{p}-\mathbf{q}\|)$;

for each singular curve $C$ of $\partial S$ do for each point $\mathbf{q}$ of $\operatorname{ProjOnCurve}(\mathbf{p}, C)$ do $D \min \leftarrow \min (D \min ,\|\mathbf{p}-\mathbf{q}\|)$;

for each superface $F$ of $S$ do for each point q of $\operatorname{ProjOnSurf}(\mathbf{p}, \operatorname{Surf}(F))$ end;

do if ClassPoint $(\mathbf{q}, S)=$ on $S$ then $\operatorname{Dmin} \leftarrow \min (\operatorname{Dmin},\|\mathbf{p}-\mathbf{q}\|)$;

Observe that we project $\mathrm{p}$ on the host surface $\operatorname{Surf}(F)$ of each superface $F$ of $S$, and then discard those points in the projection that are not on $S$ and therefore are not in $S$ 's smooth faces. ProjOnSurf is easy to implement for standard surfaces and hence for PCC approximations of canal surfaces, but projecting $\mathbf{p}$ directly on the smooth faces is non-trivial because the faces may be very complex.

ProjOnCurve is straightforward for simple curves such as lines and circles, and hence for PCCs. For a general parameterized curve $C(t)$ evaluation of the normal projection amounts to minimizing $\|p-C(t)\|$, and this can be done by setting the first derivative of $\|\mathrm{p}-C(t)\|^{2}$ to zero and solving for $t$ using a root finder.

We presented ClassPointWrtOffset and Dist as separate procedures for simplicity of exposition. But the computations may be reorganized for efficiency so as, for example, to return in $S$ as soon as a distance less than $r$ is found.

\subsubsection{Neighborhoods}

Classification procedures must return neighborhoods for the on segments to disambiguate situations wherein two solids with overlapping boundaries are combined by a Boolean operation [Tilove 80 , Requicha \& Voelcker 85]. For simplicity we ignored this fact in the discussion of algorithms above, but one must add to the returned results EonS or on $S$ a neighborhood representation with respect to $S$.

Neighborhhods for midpoints of segments in the ClassEdge procedure are essentially two-dimensional and can be represented and combined by 
standard methods [Requicha \& Voelcker 85], provided that neighborhoods with respect to offset solids are available. These can be computed as shown in Figure 8. Let $\mathbf{p}$ be a point at distance $r$ from a solid $S$, and let $\mathbf{q}_{i}$ be points of $\partial S$ where the distance is attained. Then $\mathbf{p}$ must be in the intersection of the n-offsets of the faces, singular curves and points in which the $q_{i}$ lie. Since we also know on which side of the n-offset surfaces is the solid $S \uparrow^{*} r$, it is not difficult to generate a standard neighborhood representation for $\mathbf{p}$.

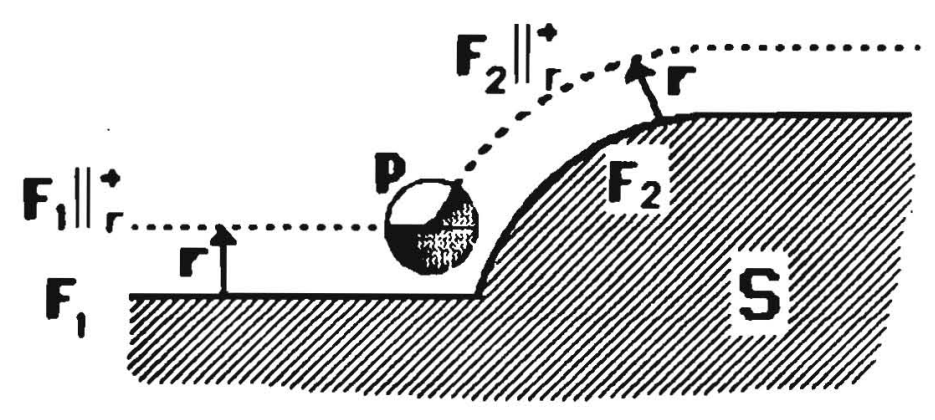

Figure 8

Computing the neighborhood of point $\mathbf{p}$ with respect to $S \uparrow r$.

Point membership classification also is used in Algorithm 7 to discard points on the surfaces of $S$ when computing the distance from p to $S$. We cannot guarantee that such points are in the interiors of edge segments of constant classification, and this leads to difficulties in neighborhood manipulation. When ambiguous on/on cases are encountered, which happens rarely in the context of distance calculations, one must either use so-called 3 -D vertex neighborhoods [Requicha \& Voelcker 85], which are rather unwieldy, or the alternative methods discussed in [Rossignac 85].

\subsection{Simplified BReps}

Boundary information normally contained in a BRep was used in the algorithms described above for only the following three purposes.

- Generate tentative faces.

- Intersect a curve with superfaces and segment the curve at the intersection points, in procedure ClassEdge.

- Compute distances to superfaces, singular curves and points. 


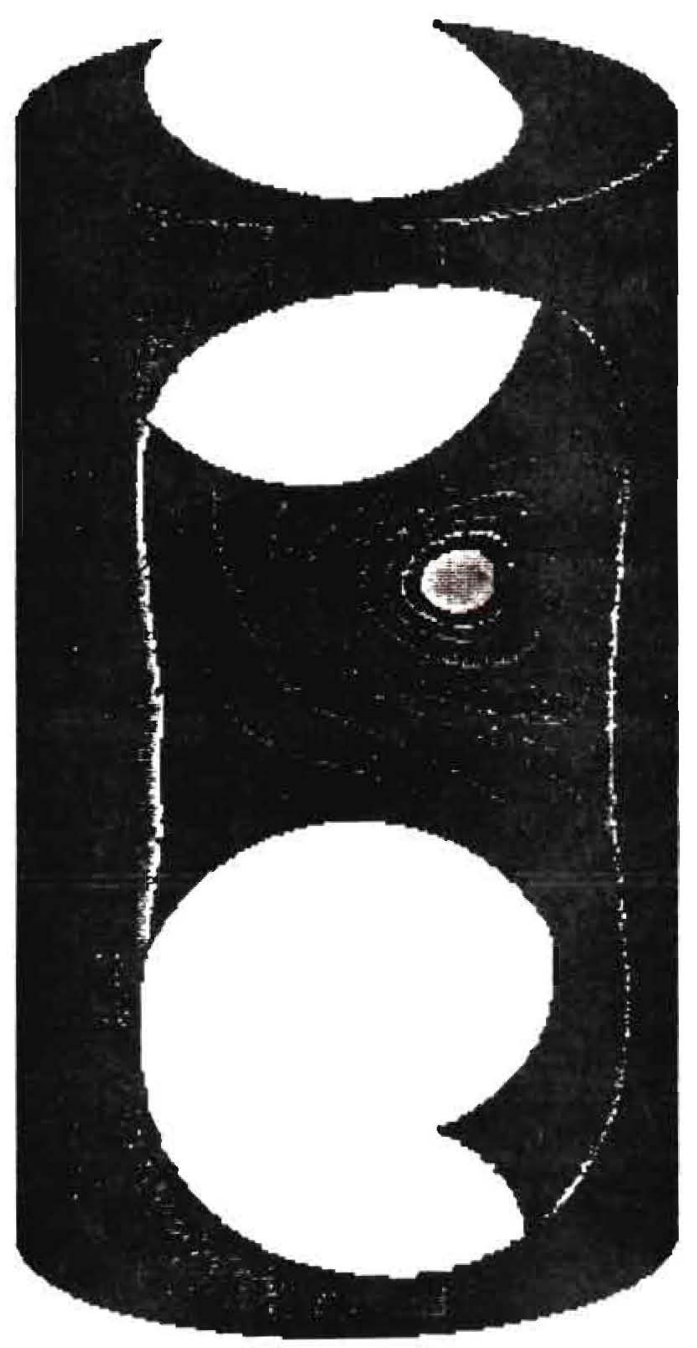

Figure 10

Shaded display generated by our experimental modeller. 


\section{APPLICATIONS}

\subsection{Blending}

We investigated in detail only one application of offsetting: the generation of constant-radius fillets and rounds, collectively called blends, in objects defined in solid modellers that support the standard surfaces [Rossignac \& Requicha 84]. Our approach is to provide facilities for defining globally blended sub-objects by using the operators $R_{r}(S)$ and $F_{r}(S)$ of Section 2.5, and to combine these blended sub-objects by Boolean operations to obtain the desired results. An example is shown in Figure 11, and details may be found in [Rossignac \& Requicha 84, Rossignac 85].

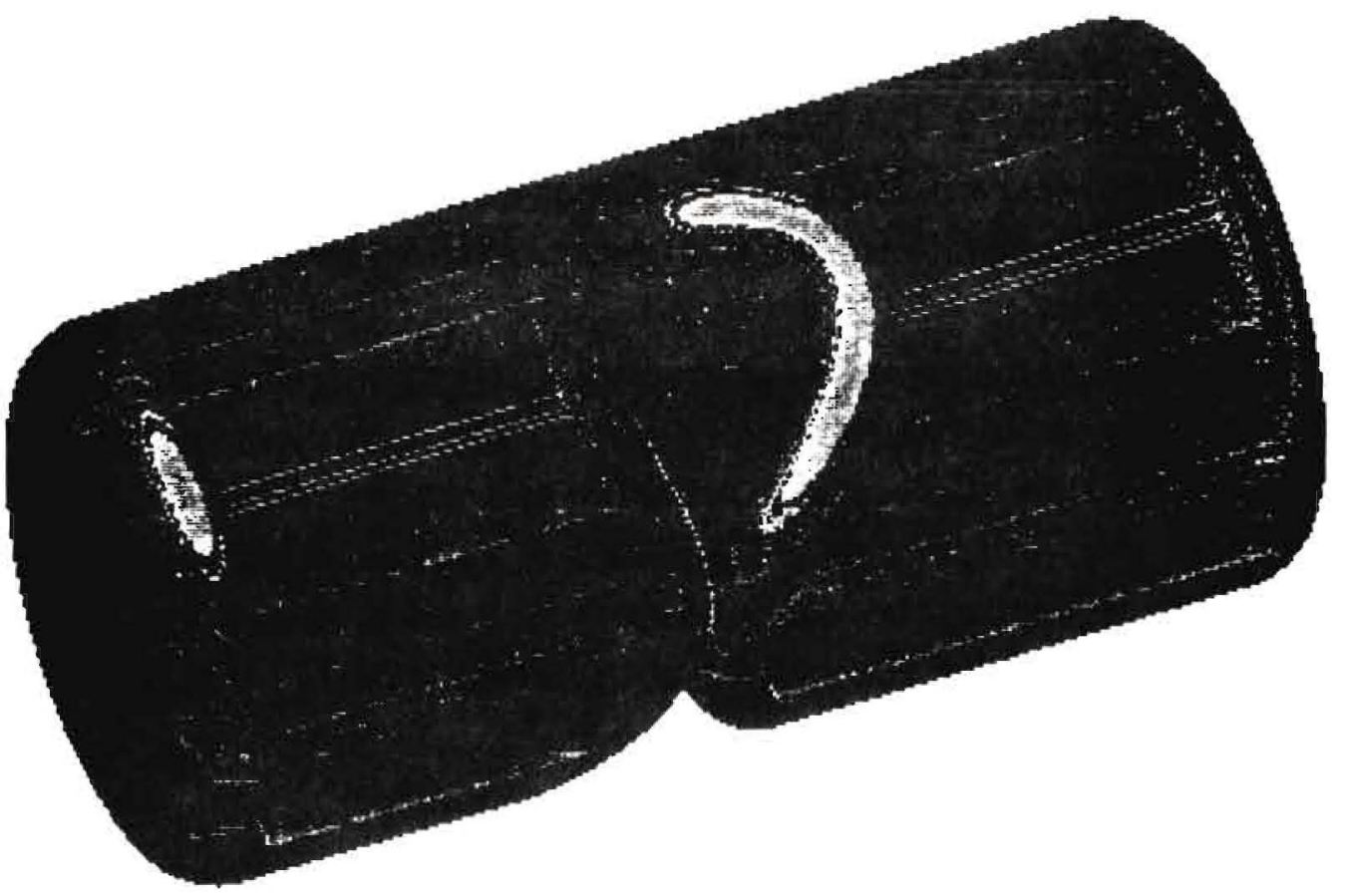

Figure 11

A blended solid. 


\subsection{Other applications}

Offsetting has many other potential applications. Examples follow.

- Tolerance analysis. Three-dimensional tolerance zones may be defined by offsets [Requicha 83], and used to determine whether mechanical parts satisfy specified tolerances.

- Approximate object equality. Two solids $X$ and $Y$ are equal within some specified resolution $\epsilon$ if $X \downarrow^{*} \epsilon \subset Y \subset X \uparrow^{*} \epsilon$. The solids $X$ and $Y$ may be, for example, a desired part and the part generated by a sequence of machining operations. Note that inclusion relations may be established through Boolean operations and null object detection [Tilove 84, Rossignac \& Voelcker 85] because, for two regular sets $X$ and $Y, X \subset Y \Longleftrightarrow X \cap^{*} Y=X$, and $X=Y \Longleftrightarrow\left(X-{ }^{*} Y\right) \bigcup^{*}\left(Y-^{*}\right.$ $X)=\emptyset$.

- Clearance tests. There is a clearance of at least $2 r$ between solids $X$ and $Y$ if $\left(X^{\uparrow^{*} r} r \bigcap^{*}\left(Y^{\uparrow^{*}} r\right)=\emptyset\right.$.

- VLSI. Clearance and overlap tests for design rule checking can be performed by using 2-D s-offsetting and Boolean operations.

- Physical process modelling. Processes such as coating and etching may be modelled, at least to a first approximation, by expanding and shrinking operations.

- Cutter path generation. Computing cutter paths for NC machining by s-offsetting solids seems more useful than current $n$-offsetting techniques, because it does not produce self-intersecting paths.

- Obstacle avoidance. Let $B$ be a ball of radius $r$ that encloses an object $X$. Then $X$ can move without colliding with obstacles $Y$ if there is a curve $C$ (traced by the center of $B$ ) such that $C \bigcap\left(Y \uparrow^{*} r\right)=\emptyset$.

\section{SUMMARY AND CONCLUSIONS}

This paper discussed a new family of operations on solids, called solid offsetting, and their incorporation in modern solid modellers that contain dual Constructive Solid Geometry (CSG) and boundary representations. Offsetting operations may be used to round and fillet solids, and have many other potential applications. 
An extended form of CSG, called CSG with offsetting (CSGO) was defined, and algorithms needed to support display generation, mass property calculation, and boundary evaluation on CSGO were presented. These algorithms use non-trivial extensions of known CSG-based methods.

Piecewise constant curvature approximations, discussed in detail elsewhere [Rossignac 85, Rossignac \& Requicha 85], were used to represent intersection edges. The resulting piecewise circular curves lead to simple and efficient procedures for computing edge/surface intersections, and distances of points to curves, which are needed by CSGO algorithms.

Experimental results show that the methods discussed are viable, but more effort is needed to improve substantially the efficiency of the algorithms. Known techniques for enhancing the average performance of geometric algorithms [Tilove 81] may hold the key to fast offsetting computations. 


\section{REFERENCES}

[Barr 84] A. H. Barr, "Global and local deformations of solid primitives", PROC. ACM SIGGRAPH '84, Minneapolis, MN, pp. 21-30, July 23-27, 1984.

[Barsky \& DeRose 84] B. A. Barsky and T. D. DeRose, "Geometric continuity of parametric curves", Report No. UCB/CSD $84 / 205$, Computer Science Dept., Univ. of California, Berkeley, October 1984.

[Barton \& Buchanan 80] E. E. Barton and I. Buchanan, "The polygon package", COMPUTER AIDED DESIGN, vol. 12, no. 1, pp. 3-11, January 1980.

[Faux \& Pratt 79] I. D. Faux and M. J. Pratt, COMPUTATIONAL GEOMETRY FOR DESIGN AND MANUFACTURE. Chichester, U.K.: Ellis Horwood Ltd., 1979.

[Foley \& van Dam 82] J. D. Foley and A. van Dam, FUNDAMENTALS OF INTERACTIVE COMPUTER GRAPHICS. Reading, MA: Addison-Wesley, 1982.

[Fournier \& Wesley 82] A. Fournier and M. A. Wesley, "Bending polyhedral objects", COMPUTER AIDED DESIGN, vol. 15, pp. 79-87, 1982.

[Hakala et al. 80] D. G. Hakala, R. C. Hillyard, P. J. Malraison and B. E. Nourse, "Natural quadrics in mechanical engineering", PROC. CAD/CAM VIII, Autofact West, Anaheim, CA, November 1980.

[Klass 83] R. Klass, "An offset spline approximation for plane cubic splines", COMPUTER AIDED DESIGN vol. 15, no. 5, pp. 297-299, September 1983.

[Lee \& Requicha 82a] Y. T. Lee and A. A. G. Requicha, "Algorithms for computing the volume and other integral properties of solids: I - Known methods and open issues", COMM. ACM, vol. 25, no. 9, pp. 635-641, Septem- 
ber 1982 .

[Lee \& Requicha 82b] Y. T. Lee and A. A. G. Requicha, "Algorithms for computing the volume and other integral properties of solids: II - A family of algorithms based on representation conversion and cellular approximation", COMM. ACM, vol. 25, no. 9, pp. 642-650, September 1982 .

[Lozano-Perez \& Wesley 79] T. Lozano-Perez and M. A. Wesley, "An algorithm for planning collision-free paths amongst polyhedral obstacles", COMM. ACM, vol. 22, no. 10, pp. 560570, October 1979.

[Matheron 75] G. Matheron, RANDOM SETS AND INTEGRAL GEOMETRY. New York: John Wiley and Sons, 1975.

[Mendelson 75] B. Mendelson, INTRODUCTION TO TOPOLOGY. Boston: Allyn and Bacon, 3rd ed., 1975.

[Monge 1849] G. Monge, APPLICATIONS DE L'ANALYSE À LA GÉOMÉTRIE. Paris: Bachelier, 5th ed., 1849.

[Nadler 78] S. B. Nadler Jr., HYPERSPACES OF SETS. New York: Marcel Dekker, 1978.

[Newman \& Sproull 79] W. M. Newman and R. F. Sproull, PRINCIPLES OF INTERACTIVE COMPUTER GRAPHICS. New York: McGraw-Hill, 2nd ed., 1979.

[Pressman \& Williams 77] R. S. Pressman and J. E. Williams, NUMERICAL CONTROL AND COMPUTER-AIDED MANUFACTURING. New York: John Wiley and Sons, 1977.

[Requicha \& Voelcker 77] A. A. G. Requicha and H. B. Voelcker, "Constructive solid geometry", Tech. Memo. No. 25, Production Automation Project, Univ. of Rochester, November 1977. 
[Requicha 77] A. A. G. Requicha, "Mathematical models of rigid solid objects", Tech. Memo. No. 28, Production Automation Project, Univ. of Rochester, November 1977.

[Requicha 80] A. A. G. Requicha, "Representations for rigid solids: theory, methods, and systems", ACM COMPUTING SURVEYS vol. 12, no. 4, pp. 437-464, December 1980 .

[Requicha \& Voelcker 82] A. A. G. Requicha and H. B. Voelcker, "Solid modelling: A historical summary and contemporary assessment", IEEE COMPUTER GRAPHICS \& APPLICATIONS, vol. 2, no. 2, pp. 9-24, March 1982.

[Requicha \& Voelcker 83] A. A. G. Requicha and H. B. Voelcker, "Solid modelling: Current status and research directions", IEEE COMPUTER GRAPHICS \& APPLICATIONS, vol. 3, no. 7, pp. 25-37, October 1983.

[Requicha 83] A. A. G. Requicha, "Toward a theory of geometric tolerancing", INTERNATIONAL JOURNAL OF ROBOTICS RESEARCH, vol. 2, no. 4, pp. 4560, Winter 1983.

[Requicha \& Voelcker 85] A. A. G. Requicha and H. B. Voelcker, "Boolean operations in solid modelling: Boundary evaluation and merging algorithms", PROC. IEEE, vol. 73, no. 1, pp. 30-44, January 1985 .

[Rossignac 85] J. R. Rossignac, "Blending and offsetting solid models", Tech. Memo. No. 54 (Ph.D. Dissertation), Production Automation Project, Univ. of Rochester, June 1985.

[Rossignac \& Requicha 84] J. R. Rossignac and A. A. G. Requicha, "Constant radius blending in solid modelling", COMPUTERS IN MECHANICAL ENGINEERING, vol. 3, no. 1, pp. 65-73, 1984. 
[Rossignac \& Requicha 85] J. R. Rossignac and A. A. G. Requicha, "Piecewise constant curvature approximation for solid modelling", Technical Memo. No. 63, in draft, Production Automation Project, Univ. of Rochester, 1985.

[Rossignac \& Voelcker 85] J. R. Rossignac and H. B. Voelcker "Redundancy and null object detection in constructive solid geometry", Technical Memo. No. 52, in draft, Production Automation Project, Univ. of Rochester, June 1985.

[Roth 82] S. D. Roth, "Ray casting for modeling solids", COMPUTER GRAPHICS AND IMAGE PROCESSING, vol. 18, no. 2, pp. 109-144, February 1982.

[Salmon 1882] G. Salmon, A TREATISE ON THE ANALYTIC GEOMETRY OF THREE DIMENSIONS. Dublin: Hodges, Figgis and Co., 4th ed., 1882.

[Serra 82] J. Serra, IMAGE ANALYSIS AND MATHEMATICAL MORPHOLOGY. New York: Academic Press, 1982.

[Shafer \& Kanade 83] S. Shafer and T. Kanade, "The theory of straight homogeneous generalized cylinders", Report CMU-CS83-105, Computer Science Dept., Carnegie-Mellon Univ., January 1983.

[Tiller \& Hanson 84] W. Tiller and E. G. Hanson, "Offsets of twodimensional profiles", IEEE COMPUTER GRAPHICS AND APPLICATIONS, vol. 4, no. 9, pp. 3646, September 1984.

[Tilove 80] R. B. Tilove, "Set membership classification: a unified approach to geometric intersection problems", IEEE TRANS. COMPUTERS, vol. C-29, no. 10, pp. 874-883, October 1980.

[Tilove 81] R. B. Tilove, "Exploiting spatial and structural locality in geometric modelling", Tech. Memo. No. 38, Production Automation Project, Univ. of 
Rochester, October 1981.

[Tilove 84] R. B Tilove, "A null-object detection algorithm for constructive solid geometry", COMM. ACM, vol. 2, no. 7, pp. 684-694, July 1984.

[Tilove et al. 84] R. B. Tilove, A. A. G. Requicha and M. R. Hopkins, "Efficient editing of solid models by exploiting structural and spatial locality", Technical Memo. No. 46, Production Automation Project, Univ. of Rochester, January 1984. (To appear in COMPUTER-AIDED GEOMETRIC DESIGN, 1985.)

[Willmore 58] T. J. Willmore, DIFFERENTIAL GEOMETRY. Oxford: Oxford University Press, 1958. 